\title{
Formation and Aggregation of Lipid Rafts in $\gamma \delta$ T Cells Following Stimulation with Mycobacterium tuberculosis Antigens
}

\author{
He-Zuo Lü, ${ }^{1,2, *}$ An-You Zhu, ${ }^{2, *}$ Yong Chen, ${ }^{2}$ Jie Tang ${ }^{2}$ and Bai-Qing $\mathrm{Li}^{2}$ \\ ${ }^{1}$ Central Laboratory, the First Affiliated Hospital of Bengbu Medical College, Anhui, P.R. China \\ ${ }^{2}$ Department of Immunology, Bengbu Medical College, and Anhui Key Laboratory of Infection and Immunity at \\ Bengbu Medical College, Anhui, P.R. China
}

\begin{abstract}
Lipid rafts are plasma membrane microdomains that are implicated in diverse signaling pathways in immune cells. Based on the distinct types of T-cell receptors, two T-cell subpopulations have been identified: $\alpha \beta$ and $\gamma \delta$ T cells. In humans, $\gamma \delta$ T cells represent a relatively rare $\mathrm{T}$ lymphocyte population but play a critical role in the immune response to infection by Mycobacterium tuberculosis. It has been demonstrated that Mycobacterium tuberculosis antigens (Mtb-Ag) preferentially activate $\gamma \delta$ T cells. Thus, we investigated whether lipid rafts are involved in the Mtb-Ag-mediated activation of $\gamma \delta$ T cells. Human peripheral blood mononuclear cells (PBMCs) were stimulated with Mtb-Ag, and expression of a lipid raft marker ganglioside GM1 (GM1) was determined by flow cytometry. The aggregation of lipid rafts was evaluated by laser confocal microscopy. Non-stimulated fresh PBMCs minimally expressed GM1 (6.55 \pm $2.01 \%$ ) and had no aggregated rafts in $\gamma \delta$ T cells. Mtb-Ag stimulation gradually increased the expression of GM1 in a time-dependent manner. At $72 \mathrm{~h}$, the majority of $\gamma \delta$ T cells expressed GM1 (88.69 $\pm 7.55 \%)$. Furthermore, accompanied with the increased expression of GM1, aggregation of lipid rafts became gradually visible in $\gamma \delta$ T cells. The aggregated rafts, however, were not evenly distributed and only occurred over a small portion of GM1-positive cells. Pretreatment with methyl- $\beta$-cyclodextrin, a cholesterol-depleting reagent, completely inhibited the Mtb-Ag-mediated aggregation of lipid rafts. These results demonstrate that lipid raft aggregation occurs in Mtb-Ag-activated $\gamma \delta$ T cells, suggesting that lipid rafts are involved in activation of $\gamma \delta$ T cells.
\end{abstract}

Keywords: Mycobacterium tuberculosis antigens; $\gamma \delta$ T cells; lipid rafts; methyl- $\beta$-cyclodextrin; ganglioside GM1 Tohoku J. Exp. Med., 2011, 223 (3), 193-198. C 2011 Tohoku University Medical Press

Lipid rafts are plasma membrane microdomains that are implicated in diverse signaling pathways in immune cells. These domains are rich in glycosphingolipids, cholesterol and certain types of proteins, such as the glycosylphosphatidylinositol-anchored proteins and lipid chainmodified proteins Src kinases, Ras, Src, and linker for activation of T cells (LAT) (Simons and Toomre 2000). It has been shown that engagement of T cell receptors (TCR) induces their physical recruitment to raft regions and that raft aggregation can regulate the initiation of TCR signal transduction (Janes et al. 1999, 2000; Alonso and Millan 2001).

In humans, T cells expressing the $\gamma \delta$ TCR represent a relatively rare $\mathrm{T}$ lymphocyte population and, particularly in peripheral blood, these cells account for only $1-5 \%$ (Haas et al. 1993). Nonetheless, the $\gamma \delta$ T cells play a critical role in the immune response to infection by Mycobacterium tuberculosis (M. tb) (Ladel et al. 1995b; Li et al. 1996). In con- trast to $\alpha \beta \mathrm{TCR}$, which can only recognize antigens as peptide fragments bound to molecules of the major histocompatibility complex (MHC), $\gamma \delta$ TCR recognizes whole proteins directly functions independently of MHC processing ( $\mathrm{Li}$ et al. 1998). Recent studies have shown that most of the ex vivo T cell-expressed $\gamma \delta$ TCRs lack CD3 delta chain which is common to the $\alpha \beta$ type (Hayes and Love 2002).

To date, the mechanism by which $\gamma \delta$ T cells recognize M.tb antigens (Mtb-Ag) has yet to be elucidated. Furthermore, the function of lipid rafts associated with Mtb-Ag-mediated activation of $\gamma \delta \mathrm{T}$ cells also remains uncharacterized. In the present study, the formation of lipid rafts and their aggregation on the surface of $\gamma \delta \mathrm{T}$ cells in response to Mtb-Ag exposure was investigated.

Received November 4, 2010; revision accepted for publication February 4, 2011. doi: 10.1620/tjem.223.193

*These authors contributed equally.

Correspondence: Bai-Qing Li, Depatment of Immunology and Ahui Key Laboratory of Infection and Immunity, Bengbu Medical College, 2600 Dong Hai Avenue, Bengbu, Anhui 233030, P.R. China.

e-mail: baiqingli@bbmc.edu.cn 


\section{Materials and Methods}

Culture of M. tb and preparation of Mtb- $\mathrm{Ag}$

Mtb-Ag was prepared according to the previous report (Boom et al. 1994). Briefly, M. $t b$ (H37Ra) was cultured in Suton medium for 4 to 6 weeks to the late $\log$ phase, and the mycobacterial cells were harvested, washed three times with normal saline, and re-suspended in two volume of ultra pure water to cell pellets, followed by heated at $120^{\circ} \mathrm{C}$ for $30 \mathrm{~min}$. Soluble Mtb-Ag was collected from supernatant of heat treated $M . t b$ cells and concentrated to $1 \mathrm{mg} / \mathrm{ml}$ before used for predominantly stimulating $\gamma \delta$ T cells.

\section{T cell culture and stimulation}

Peripheral blood samples were obtained with informed consent from healthy human donors (28 years old average; range, 20-35). All study procedures and objectives were approved by the Ethical Approval Committee of Bengbu Medical College. Peripheral blood mononuclear cells (PBMCs) were isolated from fresh heparinized blood by Ficoll-Hypaque density-gradient centrifugation and washed three times with RPMI 1640 (Gibco, Grand Island, NY, USA). Human T lymphocytes were stimulated with anti-human CD3 monoclonal antibody (CD3mAb clone OKT3, $5 \mu \mathrm{g} / \mathrm{ml}$; eBioscience, San Diego, CA, USA) or Mtb-Ag (5 $\mu \mathrm{g} / \mathrm{ml})$ in RPMI 1640 supplemented with $10 \%$ heat inactivated newborn calf serum (NCS, Hyclone, Logan, UT, USA) for $0 \mathrm{~h}, 0.5 \mathrm{~h}, 3 \mathrm{~h}, 6 \mathrm{~h}, 12 \mathrm{~h}, 24 \mathrm{~h}, 36 \mathrm{~h}, 48 \mathrm{~h}$ and 72 h at $37^{\circ} \mathrm{C}, 90 \%$ relative humidity and $5 \% \mathrm{CO}_{2}$.

\section{Disruption of membrane lipid rafts with methyl- $\beta$-cyclodextrin (MBCD)}

Cells were suspended at $1 \times 10^{6} / \mathrm{ml}$ in RPMI 1640 with $10 \mathrm{mM}$ $\mathrm{M} \beta \mathrm{CD}$ (Sigma, St. Louis, MO, USA) for $30 \mathrm{~min}$ at $37^{\circ} \mathrm{C}$ and then washed two times with RPMI 1640. Cells were resuspended in fresh RPMI 1640 with $10 \% \mathrm{NCS}$, and stimulated with CD3mAb $(5 \mu \mathrm{g} / \mathrm{ml})$ or Mtb-Ag $(5 \mu \mathrm{g} / \mathrm{ml})$ at $37^{\circ} \mathrm{C}$ for $36 \mathrm{~h}$.

\section{Flow cytometry (FCM)}

Cells were treated as described above for the indicated time periods, and then washed two times with phosphate-buffered saline (PBS). Staining was carried out on ice with fluorescein isothiocyanate (FITC)-conjugated CT-B (CT-B-FITC, $4 \mu \mathrm{g} / 10^{6}$ cells, Sigma) and phycoerythrin (PE)-conjugated anti-human $\mathrm{CD} 3 \mathrm{mAb}$ (anti-CD3-PE, $1 \mu \mathrm{g} / 10^{6}$ cells, Ancell, Bayport, MN, USA) or PE-conjugated antihuman TCR $\gamma \delta \mathrm{mAb}$ (anti-TCR $\gamma \delta$-PE, $1 \mu \mathrm{g} / 10^{6}$ cells, Becton Dickinson Technologies, San, Diego, CA, USA) in PBS supplemented with $5 \% \mathrm{NCS}$ and $0.1 \% \mathrm{NaN}_{3}$, on ice in a total volume of 50 $\mu 1$. After $30 \mathrm{~min}$, the cells were washed once, fixed in $1 \%$ paraformaldehyde (PFA, Sigma), and analyzed by flow cytometry on a FACSCalibur (Becton Dickinson) with a $488 \mathrm{~nm}$ argon excitation laser. The data were analyzed by WinMDI 2.8 software (J. Trotter, The Scripps Research Institute, La Jolla, CA, USA). The percentage of events reactive with CT-B-FITC and each PE-conjugated monoclonal antibody (anti-CD3 or anti-TCR $\gamma \delta$ ) was determined, setting thresholds with FITC $\left(0.2 \mu \mathrm{g} / 10^{6}\right.$ cells, Sigma) and isotypic control (PE-conjugated mouse IgG1, $1 \mu \mathrm{g} / 10^{6}$ cells, Becton Dickinson), respectively.

\section{Laser confocal microscopy (LCM)}

Cells were treated as described above for the indicated time periods, washed two times with phosphate-buffered saline (PBS), and then stained with anti-CD3-PE $\left(1 \mu \mathrm{g} / 10^{6}\right.$ cells, Ancell $)$ or anti-TCR $\gamma \delta$-PE $\left(1 \mu \mathrm{g} / 10^{6}\right.$ cells, Becton Dickinson) and CT-B-FITC (4 $\mu \mathrm{g} / 10^{6}$ cells, Sigma) for $30 \mathrm{~min}$ on ice. Unbound reagent was cleared by centrifugation and remaining cells were fixed with $4 \%$ PFA. The fixed cells were re-suspended in glycerine buffer $(0.5 \mathrm{~mol} / \mathrm{L}$ carbonate buffer solution with $50 \%$ glycerine, $\mathrm{pH}=9.5$ ). The cells were mixed by rapid pipetting, and a $10-\mu$ l aliquot was transferred to polyL-lysine-coated slides for imaging. The images were examined with a laser confocal microscopy (LSM 510, Carl Zeiss, Göttingen, Germany) and analyzed with LSM 5 Image Brower Software program associated with the microscope.

\section{Statistical analysis}

The paired data were analyzed by two-tailed Student's $t$-test. The data with three or more samples were analyzed by one way ANOVA followed by Student-Newman-Keuls tests of multiple comparisons. All differences were considered significant at $p<0.05$.

\section{Results}

Expression of GM1 on $\gamma \delta$ T cells activated by $M t b-A g$

PBMCs were stimulated for various times with Mtb-Ag ( $5 \mu \mathrm{g} / \mathrm{ml})$, and induced expression of GM1 was detected by FCM. As shown in Fig. 1A, non-stimulated fresh PBMCs $(0 \mathrm{~h})$ minimally expressed GM1 on $\gamma \delta \mathrm{T}$ cells $(6.55 \pm 2.01 \%)$. Stimulation with Mtb-Ag caused GM1 expression to increase gradually in a time-dependent manner. At $0.5 \mathrm{~h}$, the expression rate of GM1 on $\gamma \delta^{+} \mathrm{T}$ cells had risen to $22.73 \pm 4.34 \%$, and by $72 \mathrm{~h}$, GM1 expression was detected on the majority of $\gamma \delta \mathrm{T}$ cells $(88.69 \pm 7.55 \%)$. Similar results were observed in $\mathrm{CD}^{+} \mathrm{T}$ cells activated by CD3mAb. Fig. 1B shows the representatives of FCM dot plots generated at $0 \mathrm{~h}, 24$ and $72 \mathrm{~h}$ after stimulation with Mtb-Ag or CD3mAb.

Aggregation of lipid rafts in $\gamma \delta$ T cells activated by Mtb- $\mathrm{Ag}$

PBMCs were pretreated for 30 min with $\mathrm{M} \beta C D$ (10 $\mathrm{mM})$, and then stimulated with Mtb-Ag ( $5 \mu \mathrm{g} / \mathrm{ml})$ for $36 \mathrm{~h}$. GM1 expression and aggregation of lipid rafts on $\gamma \delta$ T cells and $\mathrm{CD}^{+}$pan-T cells was detected by LCM as described. We found that non-stimulated fresh PBMCs expressed GM1 on few T cells and appeared to lack lipid raft aggregation (control, Fig. 2A, D). PBMCs stimulated with Mtb-Ag for $36 \mathrm{~h}$ exhibited a marked increase in expression of GM1 on both $\gamma \delta \mathrm{T}$ cells and pan-T cells $(62.35 \pm 6.38 \%$ and $66.48 \pm 8.86 \%$, respectively, Fig. $2 \mathrm{G}$ ). In addition, lipid raft aggregation was observed on $\gamma \delta \mathrm{T}$ cells at this time point (Fig. 2B), but the proportion of raft aggregation was no more than $10 \%$ of the GM1-positive $\gamma \delta$ T cells $(5.89 \pm$ $1.57 \%$, Fig. 2G). The lipid raft aggregation was rarely observed in pan-T cells (Fig. 2E) and the proportion of raft aggregation in $\mathrm{CD}^{+}$cells was only $0.82 \pm 0.46 \%$ (Fig. $2 \mathrm{G}$ ). This was significantly lower than that in $\gamma \delta$ T cells $(p<$ $0.01)$. In $\mathrm{M} \beta \mathrm{CD}$-pretreated groups, the $\mathrm{Mtb}-\mathrm{Ag}$-mediated lipid raft aggregation was completely inhibited (Fig. 2C, F), but the expression of GM1 was significantly higher than that in the untreated Mtb-Ag-stimulated group $(p<0.01$, Fig. 2G). 
A

$\rightarrow$ Mtb-Ag $\cdots \cdots \mathrm{CD} 3 \mathrm{mAb}$
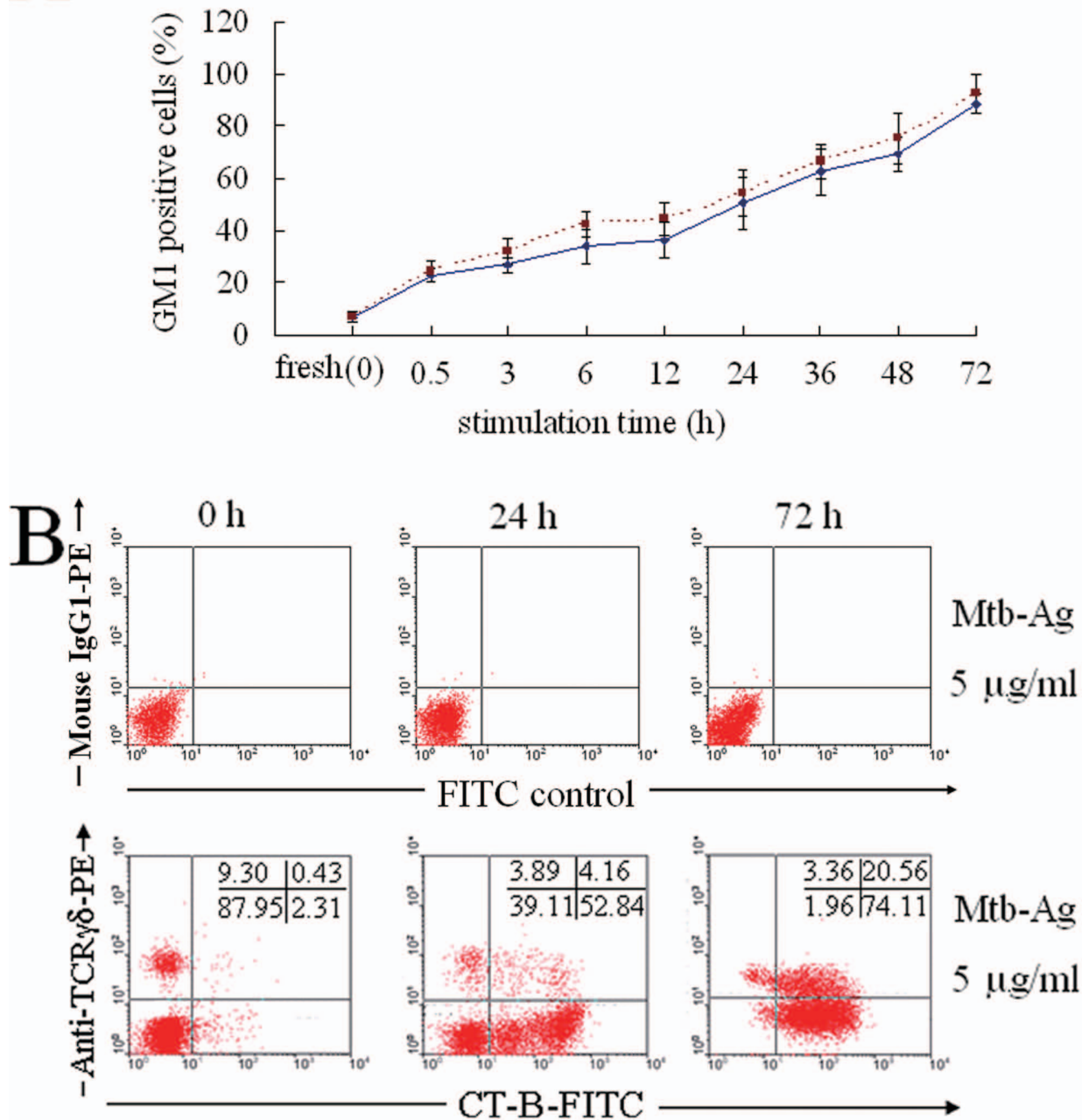

$\mathrm{Mtb}-\mathrm{Ag}$

$5 \mu \mathrm{g} / \mathrm{ml}$
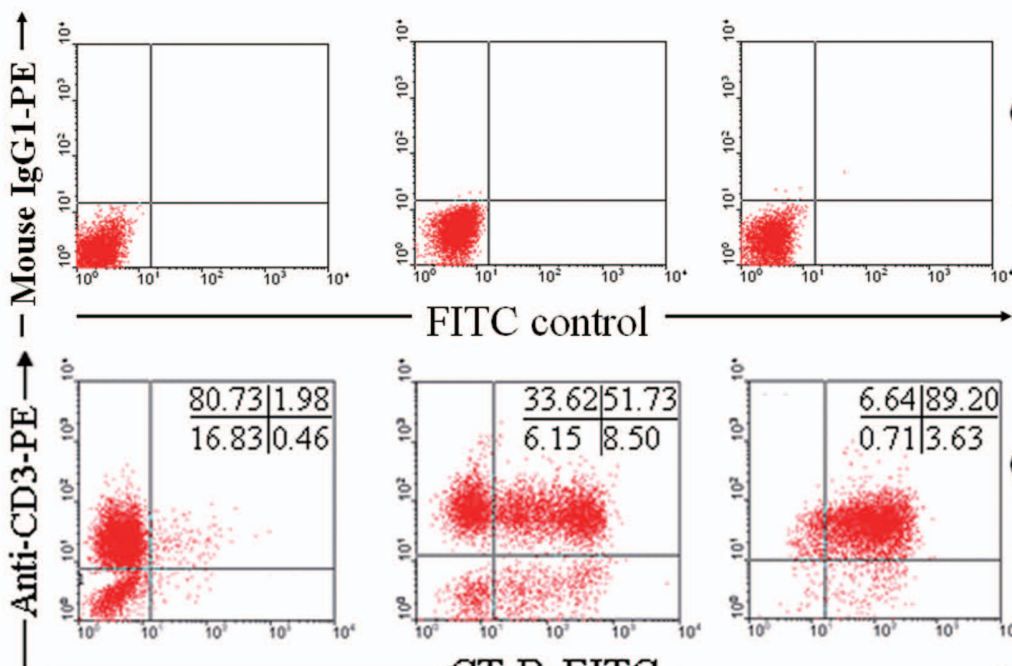

$\mathrm{CD} 3 \mathrm{mAb}$

$5 \mu \mathrm{g} / \mathrm{ml}$
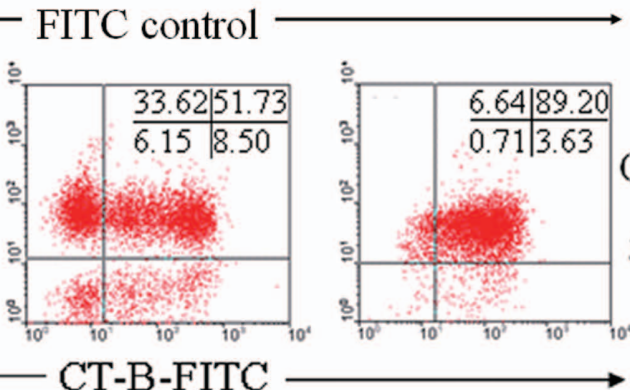

$\mathrm{CD} 3 \mathrm{mAb}$

$5 \mu \mathrm{g} / \mathrm{ml}$

Fig. 1. Expression of GM1 on Mtb-Ag-activated $\gamma \delta$ T cells.

PBMCs were incubated for different time with Mtb-Ag $(5 \mu \mathrm{g} / \mathrm{ml})$ or CD3mAb $(5 \mu \mathrm{g} / \mathrm{ml})$. The expression of GM1 on $\gamma \delta$ $\mathrm{T}$ cells and $\mathrm{CD}^{+} \mathrm{T}$ cells was detected by FCM. A: Graph showing the FCM detected expression profiles of GM1 on Mtb-Ag-activated $\gamma \delta \mathrm{T}$ cells and CD3mAb-activated $\mathrm{CD}^{+} \mathrm{T}$ cells. Data are given as means \pm standard deviation (s.D.), $n=8$. B: The profiles shown in picture are the representatives of non-stimulated fresh PBMCs $(0 \mathrm{~h})$, and Mtb-Ag or $\mathrm{CD} 3 \mathrm{mAb}$-stimulated groups ( $24 \mathrm{~h}$ and $72 \mathrm{~h}$ ). 

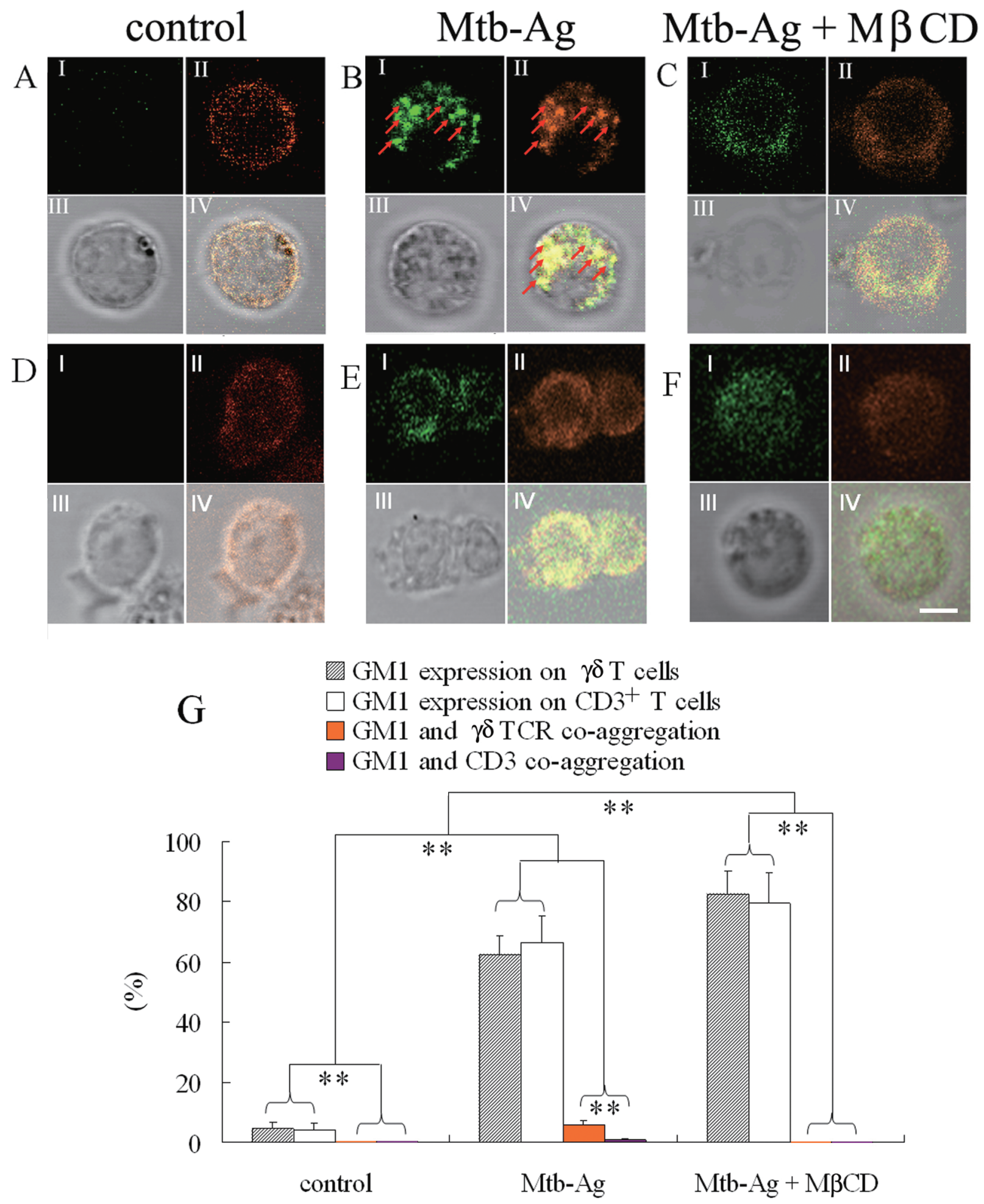

Fig. 2. Aggregation of lipid rafts in Mtb-Ag-activated $\gamma \delta$ T cells.

PBMCs were pretreated $30 \mathrm{~min}$ with $\mathrm{M} \beta \mathrm{CD}(10 \mathrm{mM})$, and then stimulated with Mtb-Ag $(5 \mu \mathrm{g} / \mathrm{ml})$ for $36 \mathrm{~h}$. The cells were labeled with CT-B-FITC and anti-TCR $\gamma \delta$-PE/ anti-CD3-PE. Aggregation of GM1 on $\gamma \delta$ T cells or CD3-positive T cells were detected by LCM. A, D: Lipid raft aggregation was undetectable in non-stimulated PBMCs (control); B, E: In Mtb-Ag-stimulated groups (Mtb-Ag), the lipid raft aggregation was visible in $\gamma \delta \mathrm{T}$ cells (B). The aggregated lipid rafts (green) and $\gamma \delta$ TCR (red) was colocalized in overlaid images (arrows in B). E: Lipid raft aggregation was very rare in $\mathrm{CD}^{+} \mathrm{T}$ cells. $\mathrm{C}$, F: In $\mathrm{M} \beta \mathrm{CD}$-pretreated groups $(\mathrm{M} \beta \mathrm{CD}+\mathrm{Mtb}-\mathrm{Ag})$, lipid raft aggregation in $\gamma \delta \mathrm{T}$ cells was inhibited (C). However, the GM1 was still highly expressed (C, F). Scale bar $=5 \mu \mathrm{m}$. G: The graph shows the statistical results. The colors of those columns that are non-visible have been labeled on the x-axis. Data are given as means \pm S.D., $n=6$, $* * p<0.01$ (ANOVA)

Note: I: CTB-FITC; II: anti-TCR $\gamma \delta$-PE in A-C, anti-CD3-PE in D-F; III: natural light; IV: overlay. 


\section{Discussion}

When different lipid species are functionally associated in biological membranes, a state of microheterogeneity is generated. Lipid rafts are microdomains that form by aggregation of glycosphingolipids and cholesterol in the plasma membrane, and on $\mathrm{T}$ cells they act to facilitate and potentiate signaling events associated with normal immune function. T cell activation in response to pathogen exposure induces a rapid aggregation of signaling molecules into reorganized rafts that are used as platforms for the assembly of a signaling complex. Aggregation of lipid rafts has been observed in the early stages of $\mathrm{T}$ cell activation following TCR engagement (Alonso and Millan 2001). GM1, a fatty acid glycosylsphingolipid, is enriched in lipid rafts, and often regarded as the signature biomarker of lipid rafts. The physical interaction of cholera toxin B subunit (CT-B) was shown by fluorescein-labeled analysis (Tuosto et al. 2001), providing a strategy, by which the distribution and aggregation of lipid rafts may be investigated.

Although $\mathrm{CD}^{+}$and $\mathrm{CD}^{+} \alpha \beta$ T cells are well recognized mediators of immunity against $M$. $t b$ infection (Kaufmann and Ladel 1994; Xing et al. 1998), recent reports from our and other labs have suggested that $\gamma \delta \mathrm{T}$ cells may also contribute to this immune response. Specifically, the principal subset of Mtb-Ag-reactive $\gamma \delta \mathrm{T}$ cell was identified as $\mathrm{V} \gamma 9 / \mathrm{V} \delta 2 \mathrm{~T}$ cells (Ladel et al. 1995a; Li et al. 1996; Dieli et al. 2000; Szereday et al. 2003). However, the mechanisms by which Mtb-Ag activates $\gamma \delta \mathrm{T}$ cells have not been fully elucidated. In order to investigate whether Mtb-Ag activates $\gamma \delta$ T cells through inducing the formation of lipid rafts, we stimulated human PBMCs with Mtb-Ag, and evaluated the expression of GM1 on $\gamma \delta$ T cells by FCM. The CD3mAb-stimulated PBMCs were used as a positive control (Schade and Levine 2002; Tomas et al. 2002; Krishnan et al. 2004). We found that GM1 was expressed highly in $\mathrm{CD}^{+} \mathrm{T}$ cells stimulated by $\mathrm{CD} 3 \mathrm{mAb}$, which was coincident with the previous reports (Schade and Levine 2002). A similar result of this study was observed in $\gamma \delta \mathrm{T}$ cells stimulated by Mtb-Ag, indicating that lipid rafts may be involved in the signal transduction process triggered by Mtb-Ag in $\gamma \delta$ T cells.

To observe the distribution and aggregation of lipid rafts on the activated T cells, we used CT-B-FITC and antiTCR $\gamma \delta$-PE/anti-CD3-PE to double-label Mtb-Ag-activated PBMCs. The changes in lipid raft morphology in $\gamma \delta \mathrm{T}$ cells and pan-T cells were visualized by LCM. We found that the lipid raft marker GM1 was detectable in both Mtb-Agactivated $\gamma \delta \mathrm{T}$ cells and $\mathrm{CD}^{+}$cells, which was coincident with the results of the GM1 expression detected by FCM. However, the proportion of aggregated rafts detected by LCM was very different between the $\gamma \delta \mathrm{T}$ cells and $\mathrm{CD}^{+}$ cells. Co-aggregation of GM1 and $\gamma \delta$ TCR could be clearly detected in overlay images. In contrast, lipid raft aggregation was very rare in pan-T cells. These results suggest that Mtb-Ag does not have the same effect on lipid raft aggrega- tion in $\gamma \delta$ T cells as it does in $\alpha \beta$ T cells.

Interestingly, although GM1 was expressed on the majority of the Mtb-Ag-activated $\gamma \delta \mathrm{T}$ cells, the proportion of lipid raft aggregation was less than $10 \%$ of GM1-positive $\gamma \delta$ cells. It is likely that GM1 is abundant in Mtb-Agactivated $\gamma \delta$ T cells and aggregation of lipid rafts is a highly dynamic process. Thus, we may only be able to detect a small fraction of co-aggregation of GM1 and $\gamma \delta$ TCR at any given time during the infection response. Among those cells with significant raft aggregation, the aggregated lipid rafts and $\gamma \delta$ TCR were co-localized.

To further confirm whether lipid rafts are involved in the Mtb-Ag-mediated activation of $\gamma \delta \mathrm{T}$ cells, we pretreated PBMCs with $\mathrm{M} \beta \mathrm{CD}$ to deplete plasma membrane cholesterol. The result showed that the Mtb-Ag-mediated raft aggregation in $\gamma \delta$ T cells was markedly inhibited by $\mathrm{M} \beta \mathrm{CD}$, but the expression of GM1 was not likewise decreased. On the contrary, GM1 expression was increased in the $\mathrm{M} \beta \mathrm{CD}$ treatment group. These results demonstrate that plasma membrane cholesterol depletion can interfere with the aggregation of lipid rafts, as previously suggested (Schade and Levine 2002). However, to our surprise, the expression of GM1 appeared to be stimulated by $\mathrm{M} \beta \mathrm{CD}$. This finding represents the first observation of such a phenomenon and further studies are required to fully understand its implications.

In conclusion, Mtb-Ag stimulation leads to high expression of the lipid raft marker GM1 on $\gamma \delta$ T cells and to aggregation of lipid rafts in $\gamma \delta$ T cells. The aggregated rafts can be disrupted by $\mathrm{M} \beta \mathrm{CD}$ exposure. These results collectively demonstrate that formation and aggregation of lipid rafts are induced during the Mtb-Ag-mediated activation of $\gamma \delta$ T cells, suggesting that lipid rafts are involved in signaling pathways related to activation of $\gamma \delta \mathrm{T}$ cells.

\section{Acknowledgments}

This work was supported by grants from National Major Science and Technology Projects for Infectious Disease (2008ZX10003011) and the Natural Science Research Program of Department of Education of Anhui Province (No. 2003KJ258).

\section{Conflict of Interest}

We have no conflict of interest.

\section{References}

Alonso, M.A. \& Millan, J. (2001) The role of lipid rafts in signalling and membrane trafficking in T lymphocytes. J. Cell Sci., 114, 3957-3965.

Boom, W.H., Balaji, K.N., Nayak, R., Tsukaguchi, K. \& Chervenak, K.A. (1994) Characterization of a 10- to 14-kilodalton proteasesensitive Mycobacterium tuberculosis H37Ra antigen that stimulates human $\gamma \delta$ T cells. Infect. Immun., 62, 5511-5518.

Dieli, F., Troye-Blomberg, M., Ivanyi, J., Fournie, J.J., Bonneville, M., Peyrat, M.A., Sireci, G. \& Salerno, A. (2000) V V $9 / \mathrm{V} \delta 2 \mathrm{~T}$ lymphocytes reduce the viability of intracellular Mycobacterium tuberculosis. Eur. J. Immunol., 30, 1512-1519.

Haas, W., Pereira, P. \& Tonegawa, S. (1993) Gamma/delta cells. 
Annu. Rev. Immunol., 11, 637-685.

Hayes, S.M. \& Love, P.E. (2002) Distinct structure and signaling potential of the $\gamma \delta$ TCR complex. Immunity, 16, 827-838.

Janes, P.W., Ley, S.C. \& Magee, A.I. (1999) Aggregation of lipid rafts accompanies signaling via the $\mathrm{T}$ cell antigen receptor. $J$. Cell. Biol., 147, 447-461.

Janes, P.W., Ley, S.C., Magee, A.I. \& Kabouridis, P.S. (2000) The role of lipid rafts in T cell antigen receptor (TCR) signalling. Semin. Immunol., 12, 23-34.

Kaufmann, S.H. \& Ladel, C.H. (1994) Role of T cell subsets in immunity against intracellular bacteria: experimental infections of knock-out mice with Listeria monocytogenes and Mycobacterium bovis BCG. Immunobiology, 191, 509-519.

Krishnan, S., Nambiar, M.P., Warke, V.G., Fisher, C.U., Mitchell, J., Delaney, N. \& Tsokos, G.C. (2004) Alterations in lipid raft composition and dynamics contribute to abnormal $\mathrm{T}$ cell responses in systemic lupus erythematosus. J. Immunol., 172, 7821-7831.

Ladel, C.H., Blum, C., Dreher, A., Reifenberg, K. \& Kaufmann, S.H. (1995a) Protective role of $\gamma \delta$ T cells and alpha/beta T cells in tuberculosis. Eur. J. Immunol., 25, 2877-2881.

Ladel, C.H., Hess, J., Daugelat, S., Mombaerts, P., Tonegawa, S. \& Kaufmann, S.H. (1995b) Contribution of $\alpha \beta$ and $\gamma \delta$ T lymphocytes to immunity against Mycobacterium bovis bacillus Calmette Guerin: studies with T cell receptor-deficient mutant mice. Eur. J. Immunol., 25, 838-846.

Li, B., Rossman, M.D., Imir, T., Oner-Eyuboglu, A.F., Lee, C.W., Biancaniello, R. \& Carding, S.R. (1996) Disease-specific changes in $\gamma \delta$ T cell repertoire and function in patients with pulmonary tuberculosis. J. Immunol., 157, 4222-4229.

Li, H., Lebedeva, M.I., Llera, A.S., Fields, B.A., Brenner, M.B. \& Mariuzza, R.A. (1998) Structure of the $\mathrm{V} \delta$ domain of a human $\gamma \delta$ T-cell antigen receptor. Nature, 391, 502-506.

Schade, A.E. \& Levine, A.D. (2002) Lipid raft heterogeneity in human peripheral blood $\mathrm{T}$ lymphoblasts: a mechanism for regulating the initiation of TCR signal transduction. $J$. Immunol., 168, 2233-2239.

Simons, K. \& Toomre, D. (2000) Lipid rafts and signal transduction. Nat. Rev. Mol. Cell Biol., 1, 31-39.

Szereday, L., Baliko, Z. \& Szekeres-Bartho, J. (2003) $\gamma / \delta$ T cell subsets in patients with active Mycobacterium tuberculosis infection and tuberculin anergy. Clin. Exp. Immunol., 131, 287-291.

Tomas, E.M., Chau, T.A. \& Madrenas, J. (2002) Clustering of a lipid-raft associated pool of ERM proteins at the immunological synapse upon $\mathrm{T}$ cell receptor or $\mathrm{CD} 28$ ligation. Immunol. Lett., 83, 143-147.

Tuosto, L., Parolini, I., Schroder, S., Sargiacomo, M., Lanzavecchia, A. \& Viola, A. (2001) Organization of plasma membrane functional rafts upon T cell activation. Eur. J. Immunol., 31, 345-349.

Xing, Z., Wang, J., Croitoru, K. \& Wakeham, J. (1998) Protection by CD4 or CD 8 T cells against pulmonary Mycobacterium bovis bacillus Calmette-Guerin infection. Infect. Immun., 66, 5537-5542. 